\title{
Flame Propagation Dependency Analysis on Confinement Geometry Shape in Hydrogen-Air-Steam Combustion Simulations
}

\author{
Tadej Holler ${ }^{1}$, Ed M.J. Komen ${ }^{2}$, Ivo Kljenak ${ }^{1}$ \\ ${ }^{1}$ Jožef Stefan Institute \\ Jamova 39, SI-1000, Ljubljana, Slovenia \\ Tadej.Holler@ijs.si; Ivo.Kljenak@ijs.si \\ ${ }^{2}$ Nuclear Research and Consultancy Group \\ Westerduinweg 3, 1755 ZG, Petten, The Netherlands \\ Komen@nrg.eu
}

\begin{abstract}
Understanding of hydrogen combustion behaviour is an important topic in nuclear safety field, since hydrogen combustion can present a serious hazard to the containment, which serves as a final barrier for release of radioactive fission products to the environment. This paper deals with the assumption that flow conditions around the ignition region can influence the flame propagation, especially to reduce the propagation in radial direction in relation to propagation in axial direction. The analysis was performed using the Computational Fluid Dynamics approach. Results, such as axial and radial flame propagation, the pressure build-up, the maximum pressure and the established flow conditions, were monitored. These results showed that the flow conditions established in mediumscale THAI experimental vessel, which has a narrower geometry surrounding the ignition region, were successfully recreated using an artificial cylindrical wall in a large-scale experimental vessel HYKA A2. Analysis of flame propagation due to changed flow conditions was then performed, focusing on axial and particularly radial flame propagation, pressure build-up and maximum pressure.
\end{abstract}

Keywords: Hydrogen, Premixed turbulent combustion, Flame speed closure model

\section{Introduction}

The production and release of hydrogen into the containment during a postulated severe accident in a Nuclear Power Plant (NPP) is an important safety issue for Light Water Reactors (LWRs) [1]. Combustion of released hydrogen may cause structural damage to the containment, which is a concrete shield building surrounding the nuclear reactor, and may compromise its function as final barrier for release of radioactive fission products to the environment. To reduce the hydrogen risk as far as possible, hydrogen mitigation systems such as Passive Auto-catalytic Recombiners (PARs) and igniters can be installed. Computer modelling is required to demonstrate the adequacy of the NPP's hydrogen risk management system. The topic received increased attention following the Three Mile Island accident in the USA back in 1979 [2] and the accident in the Japanese Fukushima Daiichi NPP in March of 2011 [3].

Computer modelling is required for the optimal design of hydrogen mitigations systems and the assessment of the accompanied residual risks of the presence of hydrogen. Complementary to the use of so-called lumped parameter codes, which model the containment as a network of control volumes with homogeneous conditions, Computational Fluid Dynamics (CFD) modelling can be used for more detailed assessment of the hydrogen risk. For that purpose, extensive validation of CFD is a prerequisite.

Results obtained from our previous validation work [4] on large-scale HYKA A2 geometry hydrogen deflagration experiments have substantially overestimated the flame propagation especially in the radial direction. The developed velocity profile results from the hydrogen deflagration experiments [5] and simulations [6] performed in THAI mediumscaled experimental vessel indicated induced convection in vertical direction and thus the increased flame propagation velocity in that direction. These profiles were interpreted to arouse due to the geometry shape around the ignition region. Therefore, a similar geometry shape was recreated for the HYKA A2 vessel. This was achieved with an artificially created cylindrically-shaped wall around the ignition region in the computational model. The dependency of flame propagation under such conditions of hydrogen deflagration using different heights of this wall was then investigated. 


\section{Experiments}

Results obtained from medium-sized experimental vessel THAI and large-scale experimental vessel HYKA A2 were used for simulations performed in the extent of this paper.

\subsection{THAI Experimental Facility}

The THAI containment test facility, shown in Figure 1 (left), is operated by Becker Technologies GmbH in Eschborn, Germany. It is a vertical cylindrical stainless steel vessel designed to withstand pressures of 20 bar at wall temperature $20^{\circ} \mathrm{C}$. The facility is measuring $9.2 \mathrm{~m}$ in height and $3.2 \mathrm{~m}$ in diameter, bringing the total volume to $60 \mathrm{~m}^{3}$. Within the framework of the hydrogen deflagration (HD) part of the OECD-NEA THAI project [5], 29 different experiments were performed, aiming to provide a deeper insight into the phenomenology of hydrogen deflagrations. In these experiments, varying initial conditions were used, by changing the initial hydrogen and steam concentrations, temperature, pressure, ignition location, and atmosphere stratification. During these experiments, all inner structures that could possibly induce turbulence were removed, except for the experimental instrumentation, which consisted of 43 thermocouples and 4 pressure transducers distributed throughout the vessel.

\subsection{HYKA A2 Experimental Facility}

The HYKA A2 experimental facility, shown in Figure 1 (right), is located on-site and operated by the Karlsruhe Institute of Technology (KIT) in Karlsruhe, Germany. This facility is similar in shape but larger than the THAI facility, measuring $9.6 \mathrm{~m}$ in height and $6.0 \mathrm{~m}$ in diameter, with a total volume of $220 \mathrm{~m}^{3}$. It is designed to withstand pressures of up to 20 bars. One experiment of hydrogen deflagration was proposed by the Jožef Stefan Institute (JSI) from Ljubljana, Slovenia, and prepared by KIT [7]. In this experiment, the mixture was ignited $1.5 \mathrm{~m}$ above the bottom of the facility and had initial conditions of pressure $1.5 \mathrm{bar}$, temperature $92{ }^{\circ} \mathrm{C}$, and hydrogen and steam concentrations 12 vol. $\%$ and $20 \mathrm{vol} . \%$, respectively. Pressure and temperature were measured at several locations throughout the facility and visual monitoring of flame propagation was also conducted using digital cameras.

The artificial wall, on Figure 1 (right), is the artificial cylindrical wall added to computational model, in order to mimic the geometry of the THAI vessel around the ignition region. This wall with the radius of $0.7 \mathrm{~m}$ was added in three different sizes; measuring $2.0 \mathrm{~m}, 2.5 \mathrm{~m}$, and $3.0 \mathrm{~m}$ from the floor level to their top, reaching $0.5 \mathrm{~m}, 1.0 \mathrm{~m}$, and $1.5 \mathrm{~m}$, respectively, above the ignition level.
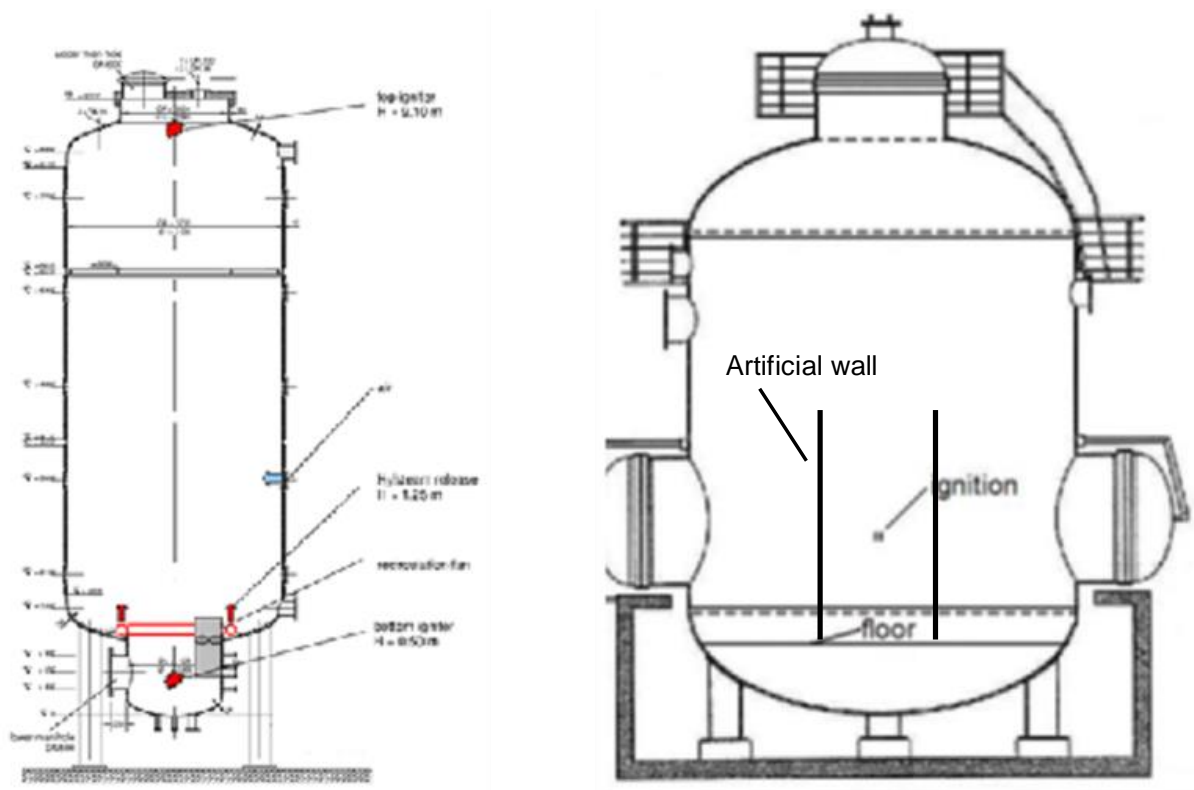

Fig. 1: Schematics of experimental vessels THAI (left) and HYKA A2 (right).

Velocity field results obtained in THAI vessel, shown on Figure 2, indicate that significant upward flow of unburned mixture is formed in the region between the flame front and the wall. This was thought to favour the axial (upward) flame 
propagation to radial (horizontal) flame propagation. Thus the idea of creating an artificial wall in HYKA A2 vessel was formed to simulate the flow conditions of THAI vessel, in order to see if previously observed overprediction of radial flame propagation [4] could be reduced.

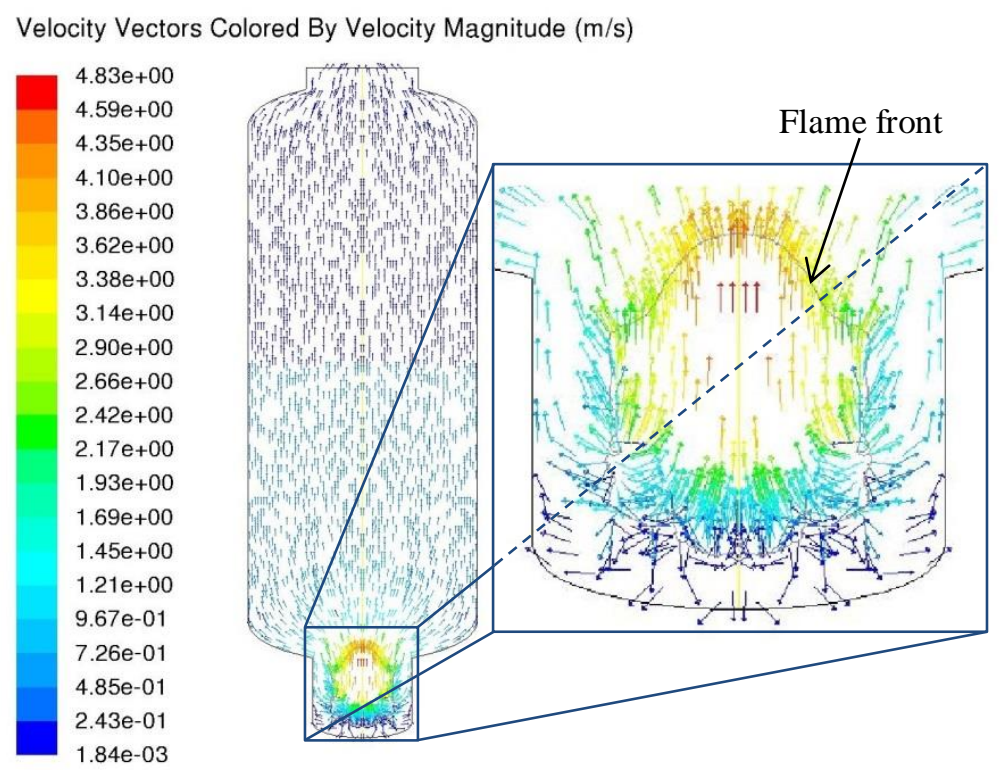

Fig. 2: Velocity field results in the initial stage of hydrogen deflagration in THAI experimental facility obtained with a simulation.

\section{Computational Approach}

The ANSYS Fluent CFD code was used as a platform to implement our hydrogen combustion model. On top of the equations for conservation of mass, momentum and energy, the additional transport equation for the Favre-averaged progress variable, $\tilde{c}$, is resolved. This progress variable is defined as:

$$
\tilde{c}=\frac{\tilde{Y}_{f}-Y_{f, u}}{Y_{f, b}-Y_{f, u}}
$$

Here $Y_{f}$ is the mass fraction of fuel, with indexes $u$ and $b$ representing the unburned and burned state, respectively.

The following paragraphs present the applied combustion model and the corresponding progress variable transport equation which has been implemented in the ANSYS Fluent code via user defined functions (UDF) for the considered simulations.

For use in weakly turbulent premixed flames, e.g. initial stages of slow deflagrations, Lipatnikov and Chomiak [8] proposed the following progress variable transport equation:

$$
\frac{\partial \bar{\rho} \tilde{c}}{\partial t}+\frac{\partial \bar{\rho} \tilde{c} \tilde{u}_{j}}{\partial x_{j}}=\frac{\partial}{\partial x_{j}}\left[\bar{\rho}\left(\kappa+D_{t}\right) \frac{\partial \tilde{c}}{\partial x_{j}}\right]+\frac{\left(S_{l}\right)^{2}}{4\left(\kappa+D_{t}\right)} \rho_{u} \tilde{c}(1-\tilde{c})+\rho_{u} U_{t}|\nabla \tilde{c}|
$$

In equation (2), $\kappa$ and $D_{t}$ are molecular and turbulent diffusivities, respectively; $\rho_{u}$ is the unburned gas density. Here $S_{l}$ represents the laminar flame speed. The third term on the right hand side of equation (2) is a quasi-laminar term. In the planar, fully developed case, the added source term results in the simple expression for the flame speed, $S_{t}=S_{l}+U_{t}$. The combustion model using this progress variable transport equation is also called Flame Speed Closure (FSC) model. Sometimes it is also referred to as the Lipatnikov combustion model. The turbulent burning velocity $U_{t}$ from equation (2) is modelled according to Zimont [9] as follows: 


$$
U_{t}=A u^{\prime} D a^{1 / 4} F(L e)
$$

Here $A$ is a model constant, $\mathrm{u}^{\prime}$ is the turbulent intensity and $D a$ is the Damkohler number. Preferential diffusion thermal (PDT) instabilities were accounted for using the $F(L e)$ term [6].

For solving the presented equations the density-based coupled solver was used in ANSYS Fluent, together with an explicit time stepping method. The turbulence model used in computations is the standard $k-\varepsilon$ model.

In order to obtain accurate results, while maintaining computational times at reasonable levels, an adaptive mesh refinement (AMR) technique was used. For the presented simulation results one level of AMR was used on top of a base grid, which was shown to be sufficient to yield reasonably grid independent results in our previous work [6]. Each level of refinement means halving the size of a computational cell in every direction of the axisymmetric 2D model used in our calculations. The base mesh cell size was around $2 \mathrm{~cm}$ by $2 \mathrm{~cm}$, while the ignition region was further refined to contain cells measured approximately $0.6 \mathrm{~mm}$ by $0.6 \mathrm{~mm}$, to keep the shape of the ignition region as even as possible. The ignition, located $1.5 \mathrm{~m}$ above the floor of the vessel, was modelled with the ignition sphere with $8 \mathrm{~cm}$ radius, to which burned properties were prescribed. The flame front position was extracted from the coordinates corresponding to the iso-surface value of $c=0.5$, as done in our previous work $[4,6,10]$. In the experiment, the reactants in the experiment were mixed using recirculation fans and then switched off several minutes prior to the ignition. Thus, the initial turbulence values were assumed to be low. However no measurements of these parameters were performed. Initial values for $k$ and $\varepsilon$ were selected as $10^{-4} \mathrm{~m}^{2} \mathrm{~s}^{-2}$ and $4.8 \cdot 10^{-5} \mathrm{~m}^{2} \mathrm{~s}^{-3}$, respectively, throughout the computational domain. This was also done in accordance with the previously conducted validations with the CFD-based modelling $[4,6,10]$. For slow deflagration computations in our previous work it was shown $[4,6,10]$, that complete heat transfer mechanism, including radiation, has a significant impact on total combustion energy output and consequently on the pressure build-up. Therefore, the simulation results were computed using prescribed constant wall temperature along with the Discrete Ordinates (DO) thermal radiation model [11]. No heat flux was prescribed to the artificial wall, with all the remaining initial and boundary conditions remaining the same as in our original analyses of hydrogen deflagration in large-scale geometry. Such rendition of the geometry resulted in exactly the same volume, bringing no additional uncertainty parameter in the mix apart from the aforementioned geometry change.

\section{Results}

In order to analyse the validity of the proposed artificial wall geometry effect on flame front propagation, axial and radial flame propagation was monitored. On top of that, the pressure build-up was also observed and all calculated variables were compared to experimental measurements. All figures show simulation results for the case with the original geometry as well as for three cases with artificial wall radius of $0.7 \mathrm{~m}$ and three different heights, i.e. $2.0 \mathrm{~m}, 2.5 \mathrm{~m}$ and $3.0 \mathrm{~m}$.
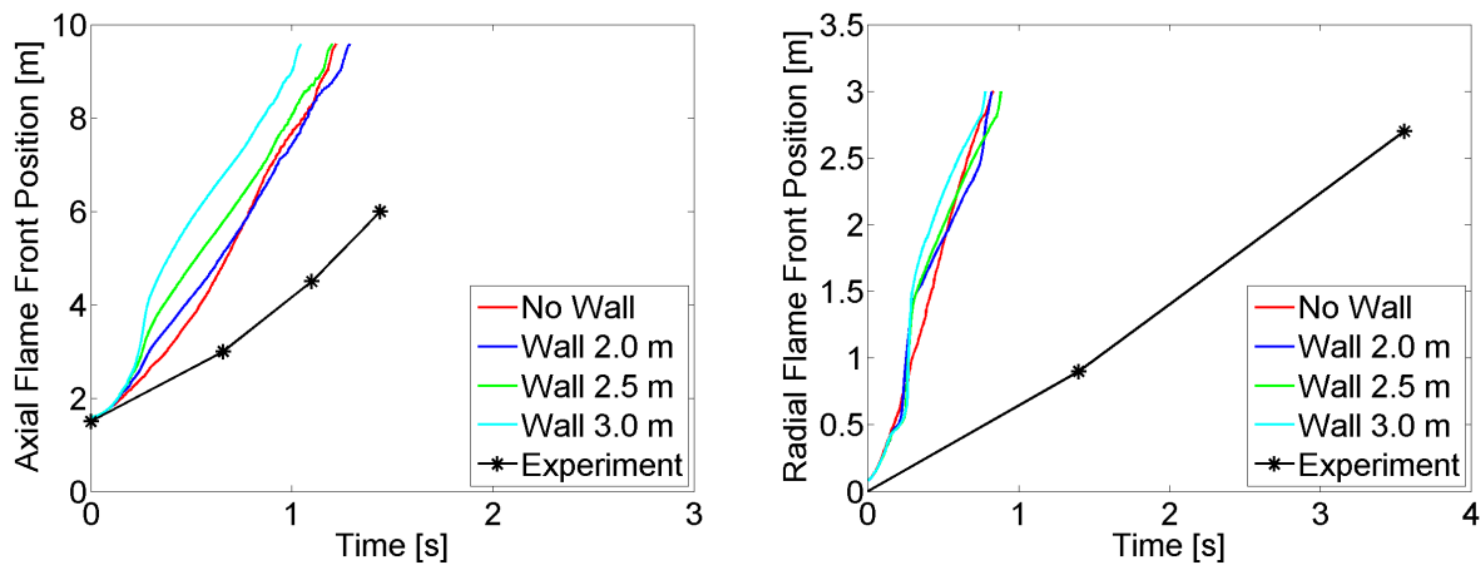

Fig. 3: Axial (left) and radial (right) flame front propagation results obtained using different heights of artificial cylindrical wall surrounding the ignition region. 
Flame front developments in both axial and radial directions on left and right parts of Figure 3 are shown, respectively. In the axial direction, a clear trend of increasing axial flame propagation rate is observed with increased wall heights, which takes the results even further from experimental measurements. In the radial direction, however, no significant flame propagation velocity decrease can be observed as a result of changed flow conditions around the ignition region. On the contrary, the flame propagates even faster in the radial direction once it comes out of the artificial cylinder.

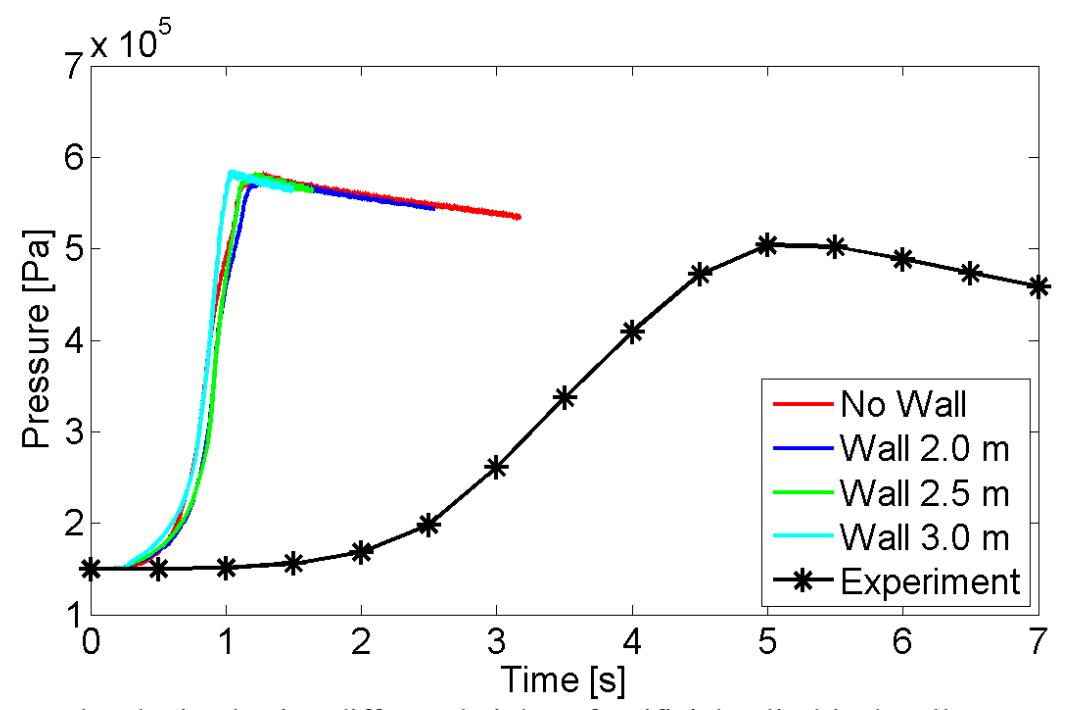

Fig. 4: Pressure history results obtained using different heights of artificial cylindrical wall surrounding the ignition region.

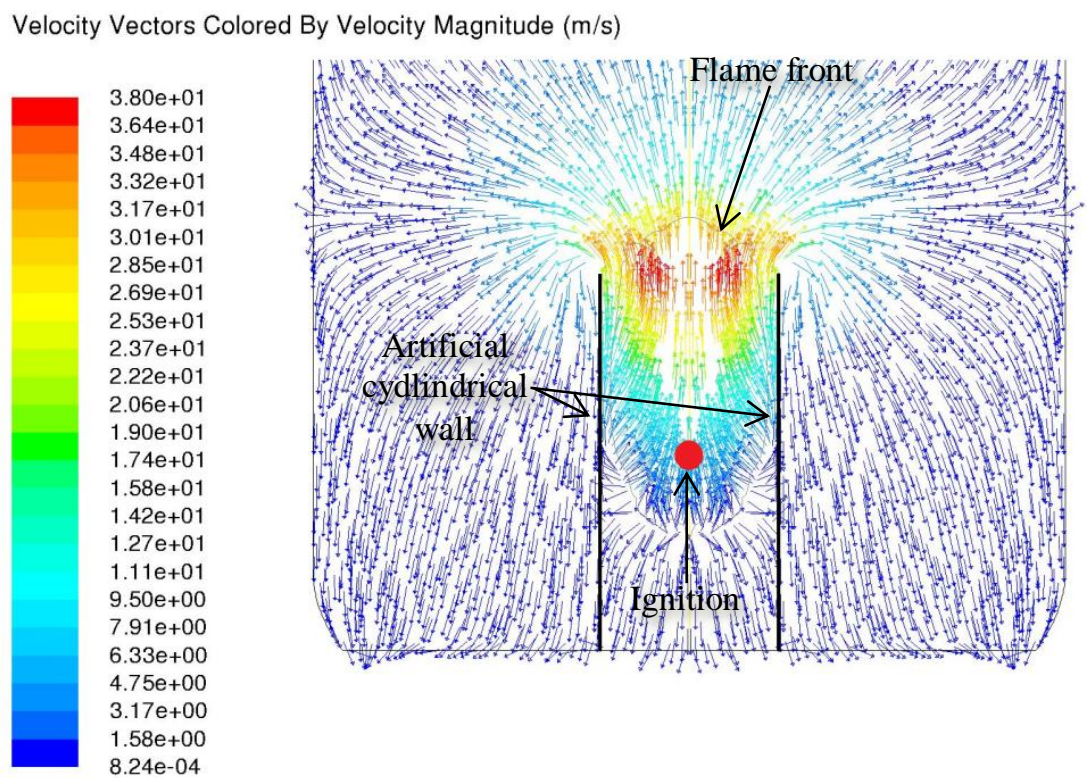

Fig. 5: Velocity field results obtained using artificial cylindrical wall with total height of $3.0 \mathrm{~m}$ surrounding the ignition region.

Looking at Figure 4, where pressure history results are plotted, no significant difference can be observed between different sizes of artificial cylindrical wall and also compared to results using the original geometry. Some very slight discrepancies can be seen only in the final stage of the pressure build-up, with maximum pressures predictions also remaining practically the same. 
Comparing all featured results with the experimental values, large discrepancies can be observed, where results obtained with simulations are substantially overpredicting both axial and radial propagation velocity as well as the pressure increase build-up. The maximum pressure is also overpredicted.

The velocity field in HYKA A2 vessel using the highest, i.e. $3.0 \mathrm{~m}$, artificial cylindrical wall, presented in Figure 5, shows similar flow conditions as observed in THAI vessel, shown in Figure 2. The velocity magnitudes in vertical direction also resemble results obtained in THAI. Here it should be mentioned, that the results obtained with different artificial wall heights are similar, and are therefore not presented in this paper.

\section{Conclusions}

This paper presents an analysis of the influence of the confinement geometry shape around the ignition region on flame propagation in hydrogen-air-steam combustion. The simulations presented here were performed with the CFD approach using the Lipatnikov combustion model. The artificial cylindrical wall was introduced to HYKA A2 in order to recreate flow conditions from the THAI vessel. This wall was tested in three different sizes.

Investigation of flow condition effects around the ignition region yielded slight increase in axial versus radial flame propagation velocity. Furthermore, once the flame front reaches the end of artificial cylindrical wall, radial flame propagation velocity actually increases. The pressure was increasing practically independently of the geometry change. This paper shows, that even though velocity field around the ignition region was successfully recreated in the HYKA A2 vessel based on THAI geometry, this flow conditions barely affect the flame propagation and do not cause slowdown of radial (horizontal) flame propagation velocity relative to propagation in axial (vertical) direction. The effects of flow conditions developed due to narrower geometry shape around ignition region can thus be proclaimed insignificant. Therefore, any further studies to determine the cause of flame front propagation overprediction in radial direction should focus on other relevant parameters, e.g. laminar flame speed.

\section{Acknowledgements}

The work described in this paper was funded by the Dutch Ministry of Economic Affairs and by the Slovenian Research Agency (ARRS) with research program "Reactor engineering" and research project "Experiment and simulation of hydrogen combustion in nuclear power plant containment experimental facility".

\section{References}

[1] I. Kljenak, A. Bentaib, and T. Jordan, "Hydrogen behavior and control in severe accidents," in: Nuclear Safety in Light Water Reactors, B. R. Sehgal ed., Elsevier, 2012, pp. 186-227.

[2] "Report of the President's Commission on The Accident at Three Mile Island", U.S. Government Printing Office, Washington D.C., USA, 1979.

[3] "Fukushima Daiichi: ANS Committee Report", American Nuclear Society Special Committee, Illinois, USA, 2012.

[4] T. Holler, V. Jain, E. Komen, and I. Kljenak, "Large-scale homogeneous hydrogen-air-steam deflagration experiment simulated using two turbulent flame speed closure models," in Proceedings of the 24th International Conference on Nuclear Engineering, Charlotte, NC, USA, 2016.

[5] T. Kanzleiter and G. Langer, "Hydrogen Deflagration Tests in the THAI Test Facility," Technical Report $1501326-$ HD-2, Becker Technologies GmbH, Eschborn, Germany, 2010.

[6] P. Sathiah, T. Holler, I. Kljenak, and E. Komen, "The role of CFD combustion modeling in hydrogen safety management-V: Validation for slow deflagrations in homogeneous hydrogen-air experiments," Nuclear Engineering and Design, 2015.

[7] I. Kljenak, M. Kuznetsov, G. Stern, M. Matkovič, and B. Mavko, "Upward Flame Propagation Experiment on Hydrogen Combustion,” Technical Report IJS-DP-11231, Jožef Stefan Institute, Ljubljana, Slovenia, 2013.

[8] A. N. Lipatnikov and J. Chomiak, "Turbulent flame speed and thickness: phenomenology, evaluation, and application in multi-dimensional simulations," Progress in Energy and Combustion Science, vol. 28, no. 1, pp. 1-74, 2002.

[9] V. L. Zimont, "Theory of turbulent combustion of a homogenous fuel mixture at high Reynolds number," Combust. Explos. Shock Waves, vol. 15, pp. 305-311, 1979. 
[10] T. Holler, V. Jain, and E. Komen, "Validation of two turbulent flame speed closure models for slow and fast hydrogen deflagration," in Proceedings of the 16th International Topical Meeting on Nuclear Reactor Thermalhydraulics: NURETH-16, Chicago, IL, USA, 2015.

[11] ANSYS-Fluent Inc., Fluent 12.0. Lebanon, NH, USA, 2008. 